\title{
Triangle Interception Scenario: A Finite-Time Guidance Approach
}

\author{
Yang Guo, ${ }^{1}$ Xiaoxiang Hu, ${ }^{1}$ Fenghua He, ${ }^{2}$ Hongjie Cheng, ${ }^{1}$ and Qinhe Gao \\ ${ }^{1}$ Xian Research Institute of High-Tech, Xian 710025, China \\ ${ }^{2}$ Control and Simulation Center, Harbin Institute of Technology, Harbin 150080, China \\ Correspondence should be addressed to Xiaoxiang Hu; huxiaoxiang2008@gmail.com
}

Received 8 October 2015; Accepted 24 December 2015

Academic Editor: Christian Circi

Copyright (c) 2016 Yang Guo et al. This is an open access article distributed under the Creative Commons Attribution License, which permits unrestricted use, distribution, and reproduction in any medium, provided the original work is properly cited.

\begin{abstract}
Considering an aircraft threatened by an interceptor, one of the effective penetration strategies is to release a Defender from the aircraft to confront the interceptor. In this case, the aircraft, the Defender, and the interceptor constitute the three-body guidance relationship, and the cooperation of the aircraft and its Defender to achieve the best tactical effects turns into a concerned problem. This paper studies the triangle interception guidance problem via the finite-time theory. The paper presents linear system InputOutput Finite-Time Stabilization (IO-FTS) method. The sufficient conditions of the linear system, being IO-FTS, under Finite-Time Boundedness (FTB) constraint are proposed, by which the state feedback controllers design method is obtained, via Linear Matrix Inequalities (LMIs). The triangle interception guidance problems are studied in three different cases, where the proposed methods are applied to the guidance design. The simulation results illustrate the effectiveness of the proposed methods.
\end{abstract}

\section{Introduction}

A triangle interception scenario emerges if the aircraft launches a Defender antimissile as an active response while facing an enemy missile. The aircraft, the enemy missile, and the Defender antimissile constitute the triangle interception engagement. In the following parts of the paper, the Evader, Pursuer, and Defender are used to refer to these three aircrafts, as shown in Figure 1. Obviously, the Evader and the Defender are on one side, the Pursuer is on the other side.

Triangle interception problems are proposed by Boyell $[1,2]$, assuming constant bearing collision courses. Using the proportional navigation (PN) guidance law, closed-form expression for the intercept point and conditions on speed ratios were also derived. PN guidance law always requires the missile to have stronger maneuver ability than the target; hence the methods did not fully exploit the position advantages of the Defender.

With the developments of missile performances and combat styles, the triangle interception problems have been a point of concern in the last several years. Ratnoo and Shima
[3] and Yamasaki and Balakrishnan [4] studied this issue; in the battle scene it was assumed that the fighter launched a Defender while facing an enemy missile. Ratnoo and Shima [3] analyzed the three-body engagement geometry; different initial conditions and guidance laws were also considered in simulation studies. Yamasaki and Balakrishnan [4] proposed a Line of Sight (LOS) guidance law for the Defender, which caused a relatively straight trajectory. The three-dimension condition was also considered in simulation study. Shaferman and Shima [5] proposed a multiple model adaptive guidance law, which had the advantages of considering detection errors and the nonlinear motion model, while it could also design cooperative guidance laws for both Defender and Pursuer.

Considering the engineering applications and classic guidance laws, LOS guidance law was concerned in triangle interception scenario. Yamasaki et al. [6] and Ratnoo and Shima [7] proposed the methods for this problem based on LOS guidance. Yamasaki et al. [6] modified the guidance laws considering different target maneuvers. Ratnoo and Shima [7] studied the strategies of Defender considering different Pursuer guidance laws. Ratnoo and Shima [8] also studied 


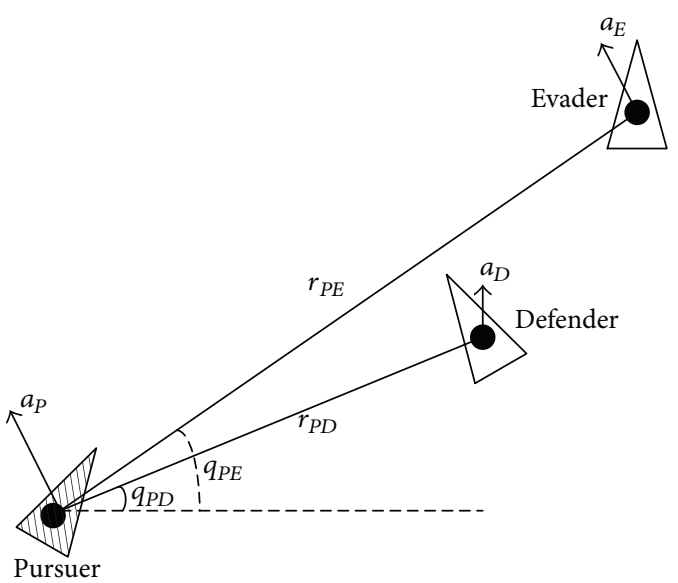

FIGURE 1: Triangle interception geometry.

Defender guidance law based on LOS, which was compared with PN guidance law. The results show that the Defender adopting the proposed method had smaller acceleration demand than the Pursuer, which would further reduce while Evader is cooperating.

Moreover, scholars considered that the three aircrafts adjust guidance strategies online instead of an invariable guidance law. Shima [9] proposed the optimal cooperative guidance law, which assumed that the interceptor adopts some linear guidance law. Rubinsky and Gutman [10] proposed performance index considering miss-distance and energy consumption simultaneously. Then the strategies of Evader and Defender were proposed based on optimal control and the influences of different time-to-go and initial conditions were analyzed. Ratnoo and Shima [11] analyzed the consequences while PN, pure pursuit, and LOS guidance laws were taken by the Pursuer and Defender. Then the boundary conditions were presented.

The concept of Finite-Time Stability (FTS) was introduced in 1960s [12]. Up until now, much work has been done in the field of FTS. Given a bound on the initial condition, a system is said to be finite-time stable if the state does not exceed a certain threshold during a specified time interval. While external disturbances are considered, FTS is extended to Finite-Time Boundedness (FTB) $[13,14]$. It worth noting that there is a different notion of FTS [15], which requires the system state to reach the system equilibrium in a finite-time interval, and the property is called finite-time attractiveness in some research $[16,17]$. In the remainder of this paper, the FTS we mentioned refers to the former one.

Input-Output Finite-Time Stability (IO-FTS) had been given in Amato et al. [18], which means that, given a class of norm bounded input signals over a specified time interval $[0, T]$, the outputs of the system are also norm bounded over $[0, T]$. Amato et al. [19-21] provided methods to solve IO-FTS problem via static state feedback and output feedback with the disturbance considered as input of the system. Therefore, this contribution can be used for disturbance suppression.
It can be concluded that FTB considers the system state (not exceeding a given threshold) in a finite-time interval while IO-FTS only considers the output (norm bounded with input disturbances satisfying some boundedness conditions) in a finite-time interval. However, in some applications, the system state stability and output should be considered comprehensively. Guo et al. [22] proposed IO finite-time stabilization method under FTB constraint for a class of linear time-invariant (LTI) systems. In terminal guidance process, if the target maneuver was considered as external disturbance, the objective of state feedback control is to minimize the impact of the disturbance to the output, and some indices satisfy the physical constraints, such as acceleration and seeker's view field of the missile. Therefore, the methods in [22] are suitable for the terminal guidance problem, but it should be further extended to linear time-variant (LTV) systems.

In the triangle interception scenario, terminal guidance processes of different flight vehicles are focused, which are all finished in finite-time intervals. The models describing the process of terminal guidance are also meaningful in some certain finite-time intervals. Moreover, the strategies of the three flight vehicles in the time interval of the Defender guidance to the Pursuer are different from the strategies of the Pursuer guidance to the Evader after escaping from the Defender. Therefore, the analysis and the guidance design of the triangle interception are typical finite-time control problem. If the infinite-time control methods were applied, the potential problems would include the closed-looped system performance with large conservative or not being able to achieve the requirements in the concerned finitetime interval. For a physical process finished in a finite-time interval, finite-time control theory may be more effective, since it only focuses on the concerned time interval and the performance indices are always defined in the concerned finite-time interval [23]. Furthermore, there have been some researches applying finite-time control theory for the terminal guidance problems in recent years [2430]. Hence, the finite-time control methods will be used to solve the triangle interception problems, as dealt with in this paper.

\section{Problem Statement}

Consider the three-body engagement scenario, as shown in Figure 1, where the Evader launches a Defender missile to intercept the incoming Pursuer.

\subsection{Assumptions}

(a) The three vehicles are constant-closing-speed point masses moving in a plane.

(b) The maneuvering dynamics of the three vehicles can be approximated by first-order transfer functions with time constants $\tau_{E}, \tau_{P}$, and $\tau_{D}$, respectively.

(c) The Pursuer adopts augmented proportional navigation (APN) guidance law. 
(d) The Evader and the Defender have the Evader acceleration information and LOS angles information between themselves and the Evader, respectively. (A triangle can be determined by three easily available parameters, which contain two LOS angles $\left(q_{P E}, q_{P D}\right)$ and one range (between Evader and Defender). Then, the Pursuer-Evader or Pursuer-Defender ranges can be determined. Furthermore, the Evader acceleration can be obtained by calculation.)

2.2. Dynamic Model. According to assumption (b), it can be obtained that

$$
\dot{a}_{i}=\frac{\left(a_{i C}-a_{i}\right)}{\tau_{i}}, \quad i=\{P, E, D\},
$$

where $a_{i}$ is acceleration and $a_{i C}$ is acceleration command. According to assumption (c), we assume that the Pursuer adopts APN guidance law, which is

$$
a_{P C}=N V_{P E} \dot{q}_{P E}+\frac{K}{2} a_{E}
$$

where $N$ and $K$ are constants.

In relative motion process between two vehicles, the rotation of the Line of Sight (LOS) satisfies

$$
\begin{aligned}
& \ddot{q}_{P E}=-2 \frac{\dot{r}_{P E}}{r_{P E}} \dot{q}_{P E}+\frac{a_{E}-a_{P}}{r_{P E}}, \\
& \ddot{q}_{P D}=-2 \frac{\dot{r}_{P D}}{r_{P D}} \dot{q}_{P D}+\frac{a_{D}-a_{P}}{r_{P D}} .
\end{aligned}
$$

$q_{P E}$ and $q_{P D}$ are LOS angles of Pursuer-Evader and PursuerDefender. Similarly, $r_{P E}$ and $r_{P D}$ are ranges of Pursuer-Evader and Pursuer-Defender. $a_{i}, i=\{P, E, D\}$ are accelerations of Pursuer, Evader, and Defender, respectively.

According to assumption (a), the three vehicles have constant-speed. Thus, after the respective collision triangles were reached and maintained, the Pursuer-Evader closing speed (denoted by $V_{P E}$ ) and that of the Pursuer-Defender (denoted by $V_{P D}$ ) are constants.

The Pursuer-Evader interception time can be approximated by

$$
t_{f P E}=\frac{r_{P E}(0)}{V_{P E}} .
$$

Similarly,

$$
t_{f P D}=\frac{r_{P D}(0)}{V_{P D}}
$$

Obviously, $t_{f P E}>t_{f P D}$ is required. For the purpose of intercepting Evader by Defender, we only consider the system behavior in the finite-time interval $\left[0, t_{f P D}\right]$.

2.3. Problem Formulation. By (1)-(5), a state space descrip-

\begin{tabular}{|c|c|c|c|c|c|c|c|}
\hline & {$[0$} & 0 & 1 & 0 & 0 & 0 & 0 \\
\hline & 0 & 0 & 0 & 1 & 0 & 0 & 0 \\
\hline & 0 & 0 & -2 & 0 & -1 & 1 & 0 \\
\hline & 0 & & $\overline{\left(t_{f P E}-t\right)}$ & 0 & $\overline{V_{P E}\left(t_{f P E}-t\right)}$ & $\overline{V_{P E}\left(t_{f P E}-t\right)}$ & U \\
\hline & 0 & 0 & 0 & -2 & -1 & 0 & 1 \\
\hline $\mathbf{A}(t)=$ & 0 & 0 & 0 & $\overline{\left(t_{f P D}-t\right)}$ & $\overline{V_{P D}\left(t_{f P D}-t\right)}$ & 0 & $\overline{V_{P D}\left(t_{f P D}-t\right)}$ \\
\hline & 0 & 0 & $\underline{N V_{P E}}$ & 0 & -1 & $K$ & 0 \\
\hline & & & $\overline{\tau_{P}}$ & & $\overline{\tau_{P}}$ & $\overline{2 \tau_{P}}$ & \\
\hline & 0 & 0 & 0 & 0 & 0 & $-\frac{1}{\tau_{E}}$ & 0 \\
\hline & 0 & 0 & 0 & 0 & 0 & 0 & $-\frac{1}{\tau_{D}}$ \\
\hline
\end{tabular}
tion can be obtained, which is

$$
\begin{aligned}
\dot{\mathbf{x}}(t) & =\mathbf{A}(t) \mathbf{x}(t)+\mathbf{B}(t) \mathbf{u}(t)+\mathbf{G}(t) \mathbf{w}(t), \\
\mathbf{y}(t) & =\mathbf{C}(t) \mathbf{x}(t),
\end{aligned}
$$

where system state vector is

$$
\mathbf{x}(t)=\left[\begin{array}{lllllll}
q_{P E} & q_{P D} & \dot{q}_{P E} & \dot{q}_{P D} & a_{P} & a_{E} & a_{D}
\end{array}\right]^{T},
$$


$\mathbf{w}(t)$ is external interference and $\mathbf{G}(t)$ is the corresponding matrix. $\mathbf{u}(t)$ and $\mathbf{B}(t)$ are defined as the following two cases.

(i) Due to the designated task of Evader, if we can only control the Defender to intercept the Pursuer, the input is $\mathbf{u}(t)=a_{D C}$; then the corresponding $\mathbf{B}(t)$ is as follows:

$$
\mathbf{B}(t)=\left[\begin{array}{lllllll}
0 & 0 & 0 & 0 & 0 & 0 & \frac{1}{\tau_{D}}
\end{array}\right]^{T} .
$$

(ii) If control authorities of both Defender and Evader are available, the input is

$$
\mathbf{u}(t)=\left[\begin{array}{ll}
a_{E C} & a_{D C}
\end{array}\right]^{T} .
$$

Then the corresponding $\mathbf{B}(t)$ is as follows:

$$
\mathbf{B}(t)=\left[\begin{array}{ccccccc}
0 & 0 & 0 & 0 & 0 & \frac{1}{\tau_{E}} & 0 \\
0 & 0 & 0 & 0 & 0 & 0 & \frac{1}{\tau_{D}}
\end{array}\right]^{T} .
$$

In this case, the acceleration of Evader is considered with a physical constraint, described as

$$
\left|a_{E}\right| \leq a_{E \max }
$$

These two cases will be discussed in Sections 4 and 5, respectively.

Define Zero-Effort-Miss distance of the Defender to the Pursuer as

$$
\mathrm{ZEM}_{P D}=\frac{\dot{q}_{P D}\left(t_{e P D}\right) D^{2}}{V_{P D}}
$$

where $t_{e P D}$ is time of Defender going in to guidance blind zone (in general, $t_{e P D}$ is near $t_{f P D}$ ) and $D$ is the size of the blind zone, a constant. Therefore, $y(t)$ is directly related to the miss-distance of the Defender to the Pursuer.

Our purpose is to get a smaller $y\left(t_{e}\right)$ by designing $\mathbf{u}(t)$. Since the system has typical finite-time characteristic, system behavior concerned only with a finite-time interval, the problem will be solved via finite-time control theory.

\section{FTB, IO-FTS, and Stabilization Method of LTV Systems}

Definition 1 ((IO-FTS of LTV systems) [18]). Given a positive scalar $T$, a positive definite matrix $\mathbf{S}_{1}$, and a positive definite matrix-valued function $\mathbf{S}_{2}(\cdot)$, system

$$
\begin{aligned}
& \dot{\mathbf{x}}(t)=\mathbf{A}(t) \mathbf{x}(t)+\mathbf{G}(t) \mathbf{w}(t), \\
& \mathbf{y}(t)=\mathbf{C}(t) \mathbf{x}(t)
\end{aligned}
$$

is said to be IO-FTS with respect to $\left(\mathrm{T}, \mathbf{S}_{1}, \mathbf{S}_{2}(\cdot)\right)$ if

$$
\int_{0}^{T} \mathbf{w}^{T}(t) \mathbf{S}_{1} \mathbf{w}(t) d t \leq 1 \Longrightarrow \mathbf{y}^{T}(t) \mathbf{S}_{2}(t) \mathbf{y}(t) \leq 1
$$

$$
\forall t \in[0, T] .
$$

Definition 2 ((FTB) [13]). Given a positive scalar $T$, a positive definite matrix $\mathbf{R}$, and a positive definite matrix-valued function $\mathbf{Q}(\cdot)$, such that $\mathbf{Q}(0)>\mathbf{R}$, system (13) and (14) is said to be Finite-Time Bounded (FTB) with respect to $\left(\mathbf{T}, \mathbf{R}, \mathbf{Q}(\cdot), \mathbf{S}_{1}\right)$, if

$$
\begin{aligned}
\mathbf{x}^{T}(0) \mathbf{R x}(0) \leq 1 \Longrightarrow \mathbf{x}^{T}(t) \mathbf{Q}(t) \mathbf{x}(t)<1 & \\
& \forall t \in[0, T]
\end{aligned}
$$

Remark 3. Compared with Lyapunov Asymptotic Stability (LAS) and BIBO stability defined in infinite-time interval, IO-FTS is only concerned with the signals defined over a finite-time interval, which is more suitable for the guidance design problems.

Both FTB and IO-FTS State Feedback Controller Design Problem. Consider a linear time-variant system (13) and (14), and a state feedback controller

$$
\mathbf{u}(t)=\mathbf{K}(t) \mathbf{x}(t),
$$

where $\mathbf{K}(t)$ is a matrix to be determined later. Applying this controller to system (13) and (14) will result in the following closed-loop system:

$$
\begin{aligned}
\dot{\mathbf{x}}(t) & =\overline{\mathbf{A}}(t) \mathbf{x}(t)+\mathbf{G}(t) \mathbf{w}(t), \\
\mathbf{y}(t) & =\mathbf{C}(t) \mathbf{x}(t),
\end{aligned}
$$

where

$$
\overline{\mathbf{A}}(t)=\mathbf{A}(t)+\mathbf{B}(t) \mathbf{K}(t) .
$$

Both FTB and IO-FTS state feedback controller design problems can be formulated as finding a state feedback controller in the form of (17) such that the closed-loop system (13) and (14) is both FTB and IO-FTS with respect to $\left(\mathbf{T}, \mathbf{R}, \mathbf{Q}(\cdot), \mathbf{S}_{1}, \mathbf{S}_{2}(\cdot)\right)$.

Theorem 4 ((necessary and sufficient conditions of IO-FTS) [20]). Given system (13) and (14) and the time interval $[0, T]$, the following statements are equivalent:

(1) System (13) and (14) is IO-FTS with respect to $\left(\mathbf{T}, \mathbf{S}_{1}, \mathbf{S}_{2}(\cdot)\right)$.

(2) The coupled DLMI/LMI

$$
\begin{aligned}
& {\left[\begin{array}{cc}
\dot{\mathbf{P}}(t)+\mathbf{A}^{T}(t) \mathbf{P}(t)+\mathbf{P}(t) \mathbf{A}(t) & \mathbf{P}(t) \mathbf{G}(t) \\
\mathbf{G}^{T}(t) \mathbf{P}(t) & -\mathbf{S}_{1}
\end{array}\right]<0,} \\
& \mathbf{P}(t) \geq \mathbf{C}^{T}(t) \mathbf{S}_{2}(t) \mathbf{C}(t), \quad t \in[0, T]
\end{aligned}
$$

$$
\text { admits a positive definite solution } \mathbf{P}(\cdot) \text { over }[0, T] \text {. }
$$

Theorem 5 (see [18]). If there exists a positive definite matrixvalued function $\mathbf{X}(\cdot)$ and a matrix-valued function $\mathbf{L}(\cdot)$ such that 


$$
\begin{aligned}
{\left[\begin{array}{cc}
-\dot{\mathbf{X}}(t)+\mathbf{X}(t) \mathbf{A}^{T}(t)+\mathbf{A}(t) \mathbf{X}(t)+\mathbf{B}(t) \mathbf{L}(t)+\mathbf{L}^{T}(t) \mathbf{B}^{T}(t) & \mathbf{G}(t) \\
\mathbf{G}^{T}(t) & -\mathbf{S}_{1}
\end{array}\right]<0, } \\
\mathbf{X}(t)^{-1} \geq \mathbf{C}^{T}(t) \mathbf{S}_{2}(t) \mathbf{C}(t), \quad t \in[0, T],
\end{aligned}
$$

the IO-FTS state feedback controller design problem can be solved by $\mathbf{K}(t)=\mathbf{L}(t) \mathbf{X}(t)^{-1}$.

Corollary 6 (both FTB and IO-FTS condition). System (13) and (14) is both FTB and IO-FTS with respect to $\left(\mathbf{T}, \mathbf{R}, \mathbf{Q}(\cdot), \mathbf{S}_{1}, \mathbf{S}_{2}(\cdot)\right)$, if there exists a symmetric positive definite matrix-valued function $\mathbf{P}(t) \in \mathfrak{R}^{n \times n}$ such that

$$
\begin{aligned}
& {\left[\begin{array}{cc}
\dot{\mathbf{P}}(t)+\mathbf{A}^{T}(t) \mathbf{P}(t)+\mathbf{P}(t) \mathbf{A}(t) & \mathbf{P}(t) \mathbf{G}(t) \\
\mathbf{G}^{T}(t) \mathbf{P}(t) & -\mathbf{S}_{1}
\end{array}\right]<0,} \\
& \mathbf{P}(t) \geq \mathbf{S}(t), \\
& \quad \text { where } \mathbf{S}(t)=\max \left(\mathbf{C}^{T}(t) \mathbf{S}_{2}(t) \mathbf{C}(t), 2 \mathbf{Q}(t)\right), \\
& \mathbf{P}(0)<\mathbf{R} .
\end{aligned}
$$

Proof. We will first demonstrate that (22), (23), and (24) can ensure that system (13) and (14) is FTB with respect to $\left(\mathbf{T}, \mathbf{R}, \mathbf{Q}(\cdot), \mathbf{S}_{1}\right)$. Let $\mathbf{V}(\mathbf{x}(t))=\mathbf{x}^{T}(t) \mathbf{P}(t) \mathbf{x}(t)$; we have

$$
\begin{aligned}
\dot{\mathbf{V}} & =\left[\begin{array}{c}
\mathbf{x}(t) \\
\mathbf{w}(t)
\end{array}\right]^{T} \\
& \cdot\left[\begin{array}{cc}
\dot{\mathbf{P}}(t)+\mathbf{A}^{T}(t) \mathbf{P}(t)+\mathbf{P}(t) \mathbf{A}(t) & \mathbf{P}(t) \mathbf{G}(t) \\
\mathbf{G}^{T}(t) \mathbf{P}(t) & 0
\end{array}\right]\left[\begin{array}{l}
\mathbf{x}(t) \\
\mathbf{w}(t)
\end{array}\right] .
\end{aligned}
$$

(In the following part of the proof, we omit $(t)$ whenever no ambiguity arises.)

By (22) and (13), we can obtain

$$
\dot{\mathbf{V}}-\mathbf{w}^{T} \mathbf{S}_{1} \mathbf{w}<0
$$

$$
\begin{aligned}
& {\left[\begin{array}{r}
\mathbf{P}(t)+\mathbf{A}^{T}(t) \mathbf{P}(t)+\mathbf{P}(t) \mathbf{A}(t)+\mathbf{B}(t) \mathbf{L}(t)+\mathbf{L}^{T}(t) \mathbf{B}^{T}(t) \\
\mathbf{P}(t) \mathbf{G}(t) \\
-\mathbf{S}_{1}
\end{array}\right] }<0, \\
& \mathbf{G}^{T}(t) \mathbf{P}(t) \mathbf{P}(t) \geq \mathbf{S}(t), \\
& \text { where } \mathbf{S}(t)=\max \left(\mathbf{C}^{T}(t) \mathbf{S}_{2}(t) \mathbf{C}(t), 2 \mathbf{Q}(t)\right), \\
& \mathbf{P}(0) \geq \mathbf{R}
\end{aligned}
$$


TABLE 1: Simulation parameters of noncooperative case.

(a)

\begin{tabular}{cccccccc}
\hline$N$ & $K$ & $V_{P E}$ & $V_{P D}$ & $t_{f P E}$ & $t_{f P D}$ & $t_{e P D}$ & $\tau_{P}$ \\
\hline 4 & 2 & $500 \mathrm{~m} / \mathrm{s}$ & $1200 \mathrm{~m} / \mathrm{s}$ & $24 \mathrm{~s}$ & $10 \mathrm{~s}$ & $9.95 \mathrm{~s}$ & $0.05 \mathrm{~s}$ \\
\hline
\end{tabular}

(b)

\begin{tabular}{lcccccc}
\hline$\tau_{D}$ & $\tau_{E}$ & $T$ & G & \\
\hline $0.05 \mathrm{~s}$ & $0.1 \mathrm{~s}$ & $9.95 \mathrm{~s}$ & {$\left[\begin{array}{lllllll}0 & 0 & 0.02 & 0.02 & 0 & 0 & 0\end{array}\right]^{T}$} \\
\hline
\end{tabular}

(c)

\begin{tabular}{lllllllll}
\hline $\mathbf{S}_{z}(\cdot)$ & $\mathbf{S}_{w}$ & $\mathbf{x}(0)$ \\
\hline $1000 t \cdot \mathbf{I}_{1 \times 1}$ & $\mathbf{I}_{1 \times 1}$ & {$\left[\begin{array}{llllllll}0.52 \pi & 0.52 \pi & -0.02 & -0.024 & 0 & 0 & 0\end{array}\right]^{T}$} \\
\hline
\end{tabular}

then both FTB and IO-FTS state feedback controller design problem can be solved by $\mathbf{K}(t)=\mathbf{L}(t) \mathbf{P}^{-1}(t)$.

Proof. Replacing $\mathbf{A}(t)$ in (13) with $\overline{\mathbf{A}}(t)$, let $\mathbf{K}(t)=\mathbf{L}(t) \mathbf{P}(t)$. According to Corollary 6 , then the proof follows.

\section{Defender Intercepts Pursuer without Cooperation of Evader (Case i)}

In some applications, an Evader has its own mission or target, which means that its trajectory will not change whether Pursuer exists or not. The state feedback is used to design controller with the object of minimizing the impact of the disturbance to the output (finite-time disturbance suppression). The acceleration command of Defender can be obtained by the following expression:

$$
a_{D C}=\mathbf{K}(t) \mathbf{x}(t),
$$

where $\mathbf{K}(t)$ can be calculated by using IO-FTS state feedback controller design method in Theorem 5.

According to the model in Section 2 and the proposed method in Section 3, using the parameters in Table 1, the controller can be obtained. Then the system performance and the trajectories of the aircrafts can be calculated, as shown in Figures 2-4.

In the noncooperation case, the Evader follows a vertical upward flight path, the Pursuer guidance to the Evader. Meanwhile, the Defender is released from the Evader and guidance to the Pursuer. Compared with the PN guidance law, the Defender trajectory caused by the proposed method is more flat without significant turns, which means that the acceleration demands of the Defender are much smaller.

Figures 3 and 4 present the LOS angular rate and the Defender acceleration. It can be found that both methods can drive the LOS angular velocity tending to zero, but the acceleration demand by the proposed method is much smaller.

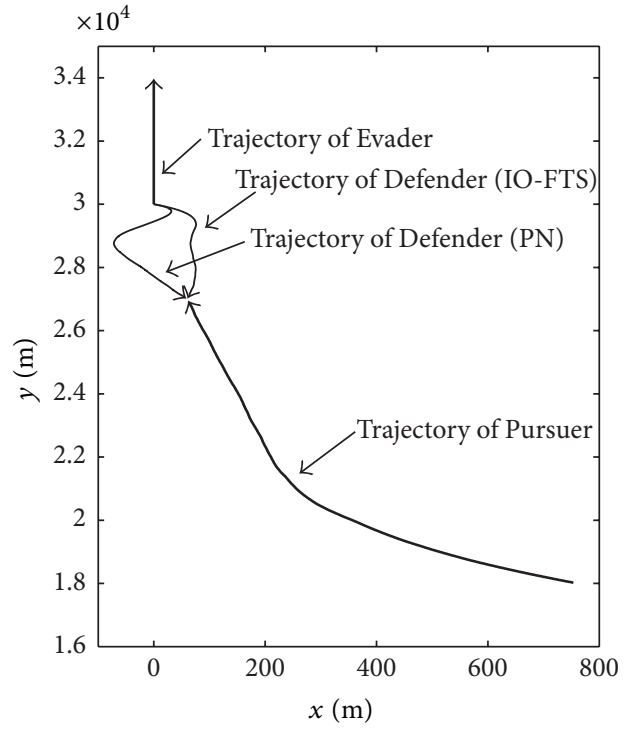

FIGURE 2: The trajectories while the Defender adopts PN or IO-FTS guidance law.

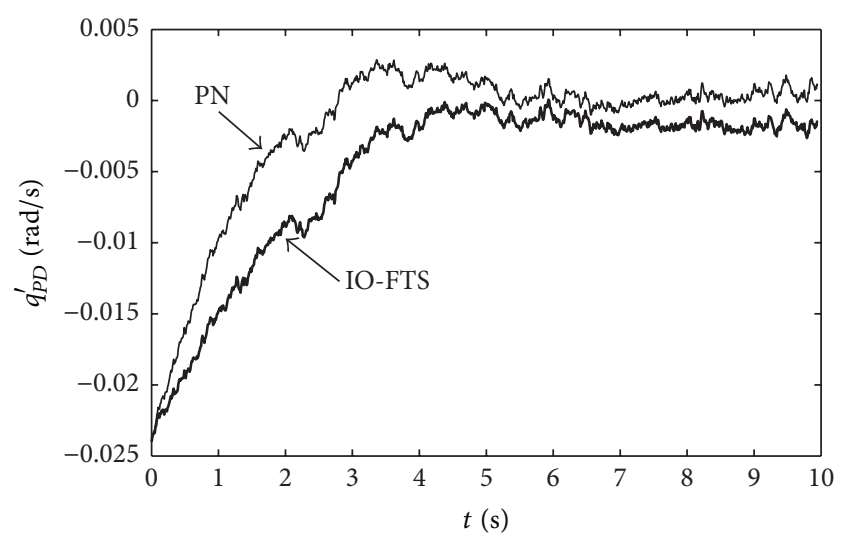

FIgURE 3: The LOS angular rate.

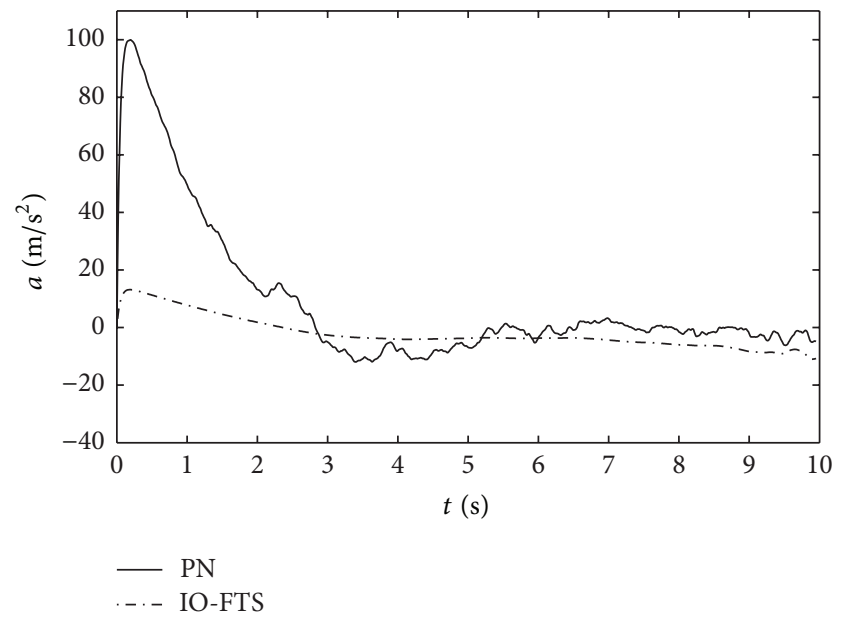

Figure 4: The Defender acceleration. 
TABLE 2: Simulation parameters of face-to-face interception case.

(a)

\begin{tabular}{lcclllllll}
\hline$t_{f P E}$ & $t_{f P D}$ & $t_{e P D}$ & $\mathbf{x}(0)$ \\
\hline $15 \mathrm{~s}$ & $10 \mathrm{~s}$ & $9.95 \mathrm{~s}$ & {$\left[\begin{array}{llllllll}0.99 \pi & 0.99 \pi & 0.04 & 0.04 & 0 & 0 & 0\end{array}\right]^{T}$} \\
\hline
\end{tabular}

(b)

\begin{tabular}{lcc}
\hline$a_{E \max }$ & $\mathbf{R}$ & $\mathbf{Q}(\cdot)$ \\
\hline $30 \mathrm{~m} / \mathrm{s}^{2}$ & $\mathbf{I}_{7 \times 7}$ & $10^{-5} \cdot \operatorname{diag}\{1,1,1,1,1,100,1\}$ \\
\hline
\end{tabular}

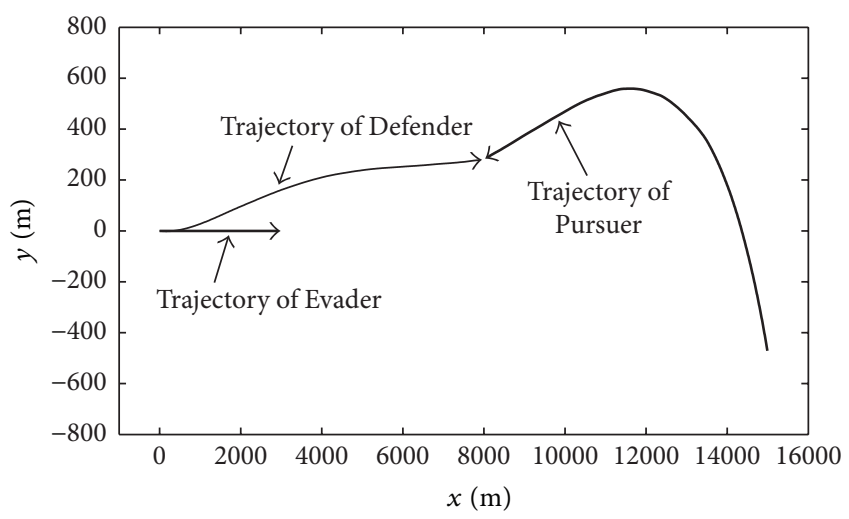

FIGURE 5: The trajectories of the noncooperative case.

\section{Evader and Defender Cooperate to Intercept Pursuer (Case ii)}

While considering a cooperation case, the Evader can "lure" the Pursuer to help the Defender achieve an easier interception. However, the acceleration physical constraint of the Evader should be considered as presented in (11). So both the disturbance depression and finite-time state bounded should be satisfied comprehensively. We proposed both FTB and IO-FTS controller design method (Corollary 7) to solve the problem.

According to the model in Section 2 and the proposed method in Section 3, using the parameters in Table 2, the controller can be obtained; then in the cases of cooperating and noncooperating Evaders, the system performances and the trajectories of the aircrafts can be calculated, as shown in Figures 5 and 6.

In the cooperative case, by mean of the Evader maneuvering to "lure" the Pursuer, the Defender more easily obtains better guidance effect. Figures 7-10 show the comparison of LOS angular rate and acceleration. In cooperative and noncooperative cases, the proposed method can drive the LOS angular rate which tends to zero, which means a small miss-distance. However, in the cooperative case, the Defender requires smaller acceleration, which can be seen in the fact that the Evader "shares" some acceleration demand.

For some high speed targets, such as reentering ballistic missiles, a head-on interception strategy will cause high closing speed. This imposes severe requirements on the interceptor systems such as precise detection of the target from a large distance by the onboard seekers and very fast

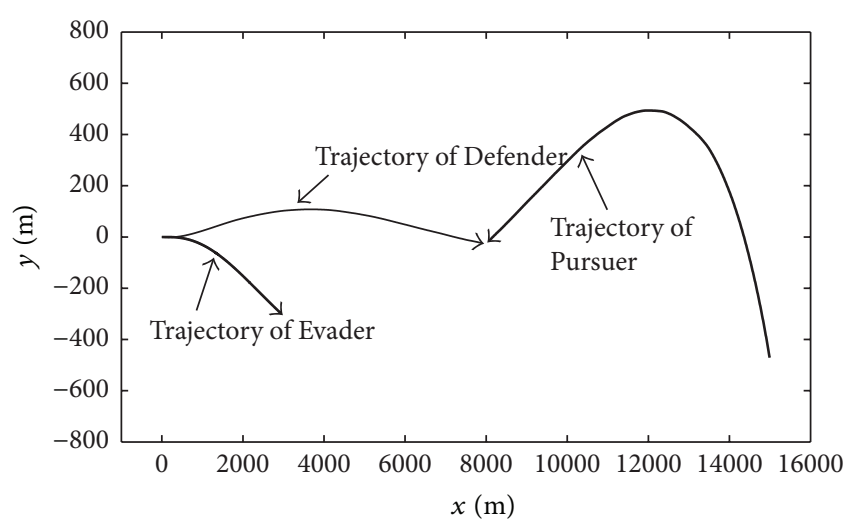

Figure 6: The trajectories of the cooperative case.

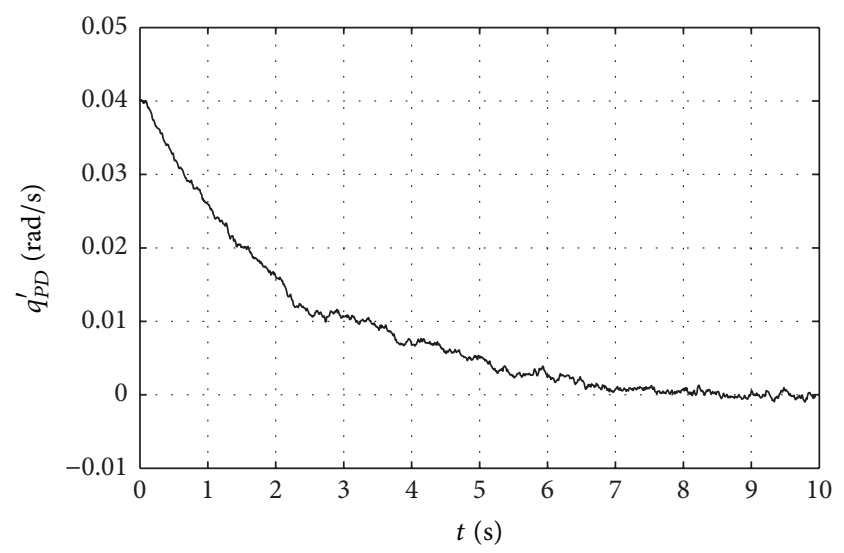

FIGURE 7: The LOS angular rate of cooperative case.

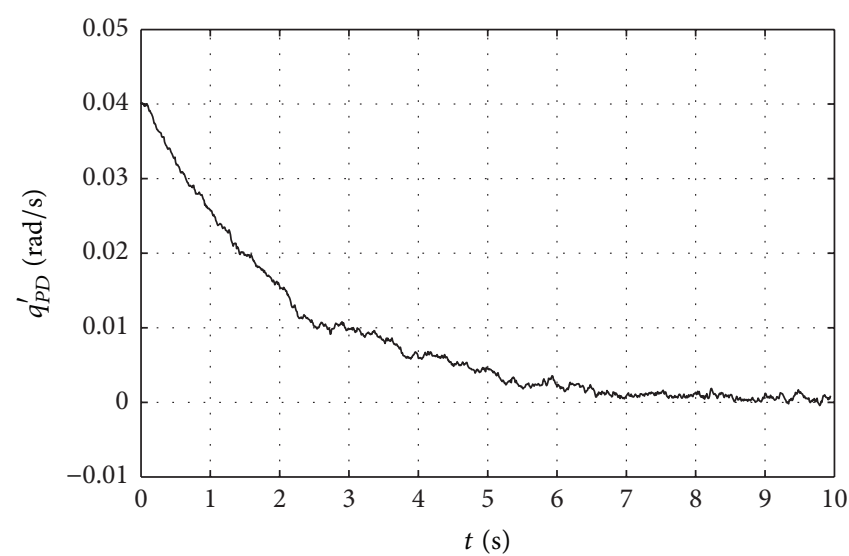

FIgURE 8: The LOS angular rate of noncooperative case.

response time of the missile subsystems. Head Pursuit (HP) guidance was introduced to solve this problem. The guidance law imposes a geometric relation in which the interceptor missile is positioned ahead of the target so that both fly in the same direction. The missile speed is planned to be lower than that of the target, and therefore the target closes on the interceptor missile [31, 32].

In our scenario, considering HP guidance adopted by the Pursuer to intercept the Evader, both FTB and IO-FTS 


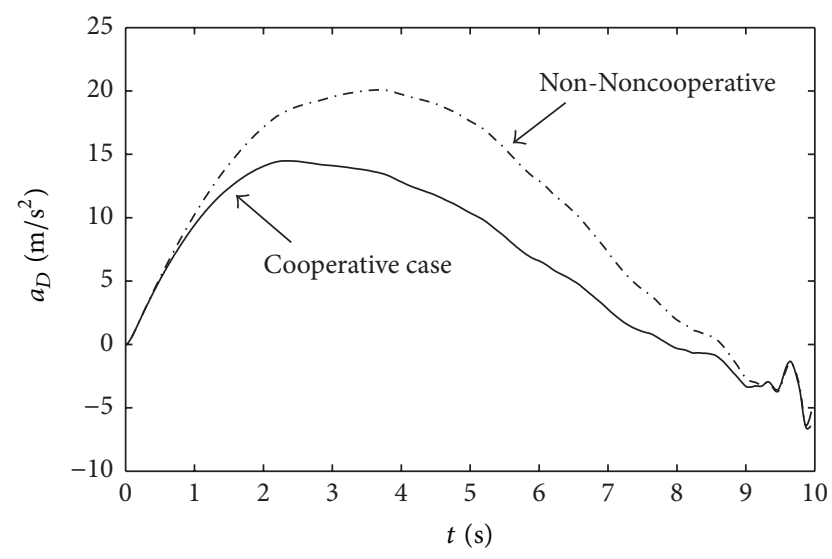

Figure 9: The Defender acceleration.

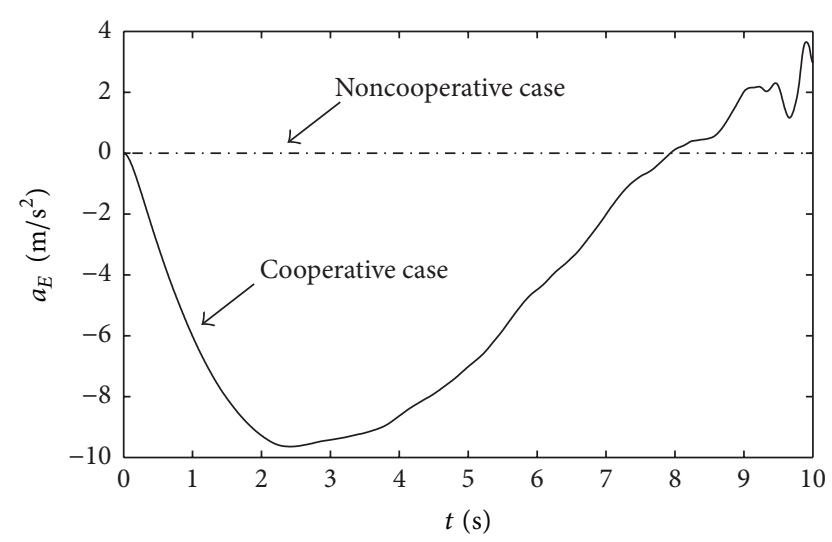

FIgURE 10: The Evader acceleration.

TABLE 3: Simulation parameters of HP guidance pursuer case.

(a)

\begin{tabular}{lllllllll}
\hline$t_{f P E}$ & $t_{f P D}$ & $t_{e P D}$ & $\mathbf{x}(0)$ \\
\hline $30 \mathrm{~s}$ & $20 \mathrm{~s}$ & $19.9 \mathrm{~s}$ & {$\left[\begin{array}{lllllll}0.997 \pi & 0.997 \pi & 0.023 & 0.02 & 0 & 0 & 0\end{array}\right]^{T}$} \\
\hline
\end{tabular}

(b)

\begin{tabular}{lcccc}
\hline$V_{P E}$ & $V_{P D}$ & $a_{E \max }$ & $\mathbf{R}$ & $\mathbf{Q}(\cdot)$ \\
\hline $500 \mathrm{~m} / \mathrm{s}^{2}$ & $750 \mathrm{~m} / \mathrm{s}^{2}$ & $30 \mathrm{~m} / \mathrm{s}^{2}$ & $\mathbf{I}_{7 \times 7}$ & $10^{-5} \cdot \operatorname{diag}\{1,1,1,1,1,100,1\}$ \\
\hline
\end{tabular}

controller design method (Corollary 7) still can be used to solve the problem. The parameters are presented in Table 3 . Then, in the cases of cooperating and noncooperating Evaders, the system performances and the trajectories of the aircrafts can be calculated, as shown in Figures 11-14.

It can be concluded that, considering HP guidance Pursuer, the closing speed between Evader and Pursuer is

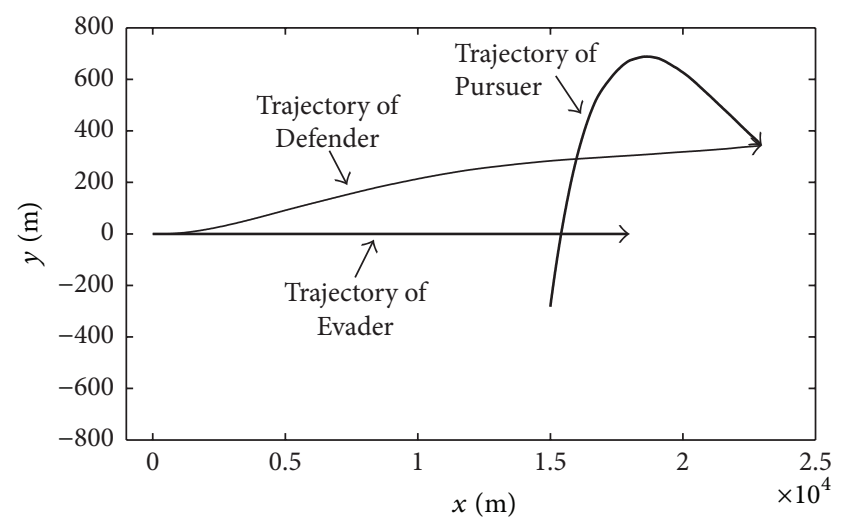

FIGURE 11: The trajectories of the noncooperative case considering HP guidance Pursuer.

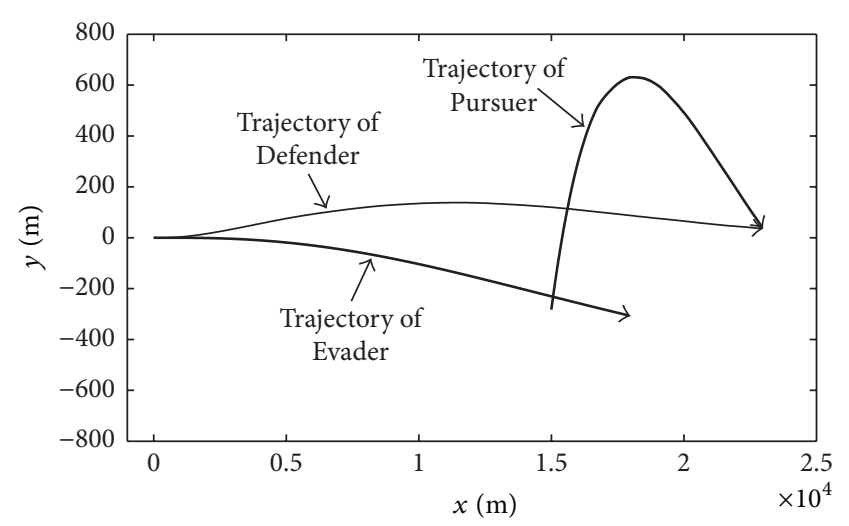

FIGURE 12: The trajectories of the cooperative case considering HP guidance Pursuer.

smaller than the PN guidance Pursuer case. With Evader and Defender cooperating, the acceleration demand of Defender is smaller than the noncooperative case.

\section{Multi-Defender versus Multi-Pursuer Case}

In this case, we consider that, while facing multiple Pursuers, an Evader launches the corresponding number of Defenders as countermeasures. Figure 15 presents the two Defenders versus two Pursuers case. On one hand, if the Evader did not maneuver cooperatively, each Defender should be guided to the designated Pursuer. The guidance law of each Defender can be designed according to Case i in Section 4 (Defender intercepts Pursuer without cooperation of Evader). On the other hand, if the Evader cooperates with the Defenders, the Evader maneuvering would influence both Defender's guidance processes.

For this problem, we define

$$
\begin{aligned}
& \mathbf{x}(t)=\left[\begin{array}{lllllllllllll}
q_{P E 1} & q_{P D 1} & \dot{q}_{P E 1} & \dot{q}_{P D 1} & q_{P E 2} & q_{P D 2} & \dot{q}_{P E 2} & \dot{q}_{P D 2} & a_{P 1} & a_{D 1} & a_{P 2} & a_{D 2} & a_{E}
\end{array}\right]^{T}, \\
& \mathbf{u}(t)=\left[\begin{array}{lll}
a_{E C} & a_{D C 1} & a_{D C 2}
\end{array}\right]^{T}, \\
& \mathbf{y}(t)=\left[\begin{array}{ll}
\dot{q}_{P D 1} & \dot{q}_{P D 2}
\end{array}\right]^{T} \text {. }
\end{aligned}
$$




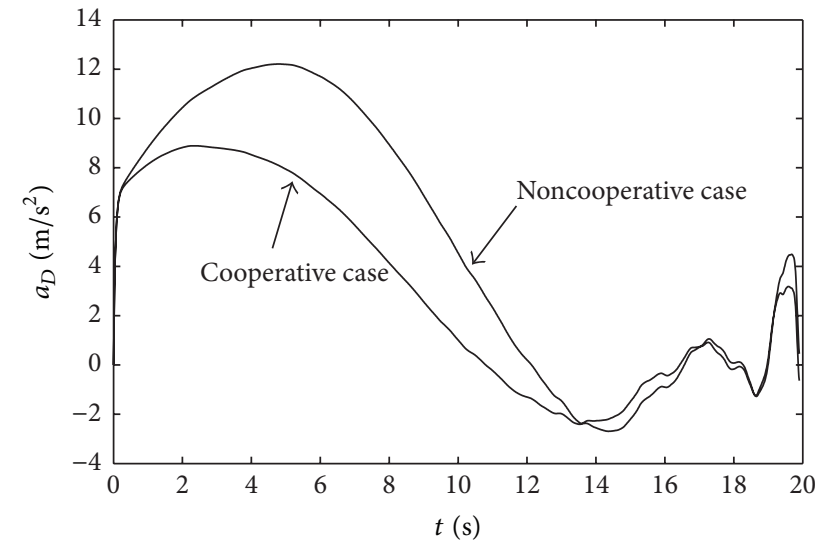

FIgURE 13: The Defender acceleration considering HP guidance Pursuer.

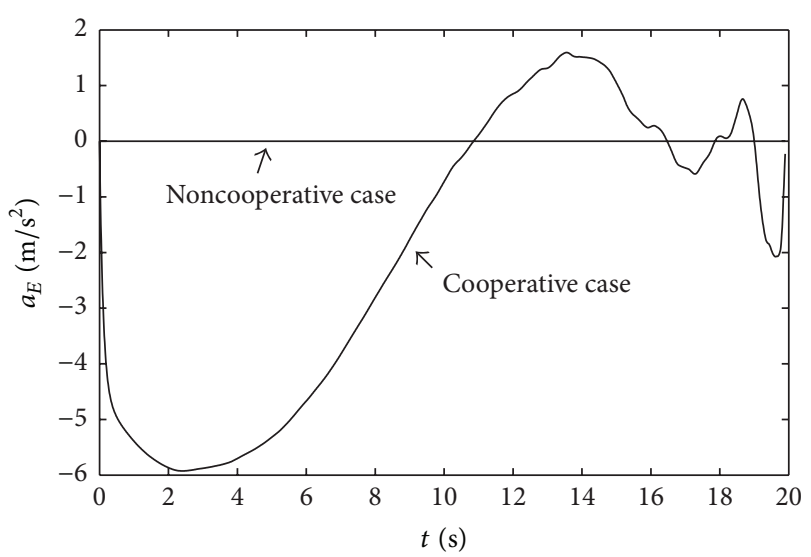

FIgURE 14: The Evader acceleration considering HP guidance Pursuer.

According to the modeling method in Section 2, we can get the similar system as follows:

$$
\begin{aligned}
& \dot{\mathbf{x}}(t)=\mathbf{A}(t) \mathbf{x}(t)+\mathbf{B}(t) \mathbf{u}(t)+\mathbf{G}(t) \mathbf{w}(t), \\
& \mathbf{y}(t)=\mathbf{C}(t) \mathbf{x}(t)
\end{aligned}
$$

$\mathbf{A}(t), \mathbf{B}(t)$, and $\mathbf{G}(t)$ can be obtained by similar equations in Section 2. It is worth noting that the two Defender guidance processes are likely finished at different moments. We use $t_{f P D 1}, t_{f P D 2}$ to denote the guidance finish moment of the two Evaders, respectively. Then, in time interval $\left[\min \left\{t_{f P D 1}, t_{f P D 2}\right\}, \max \left\{t_{f P D 1}, t_{f P D 2}\right\}\right]$, one Defender guidance process has finished; we focus on the remaining three flight vehicles, so the problem turns into the same one in Section 5 . In time interval $\left[0, \min \left\{t_{f P D 1}, t_{f P D 2}\right\}\right]$, we focus on all five flight vehicles' guidance processes.

In the time interval $\left[0, \min \left\{t_{f P D 1}, t_{f P D 2}\right\}\right]$, we still can solve guidance design problems according to the method in Corollary 7. The difference is that $\mathbf{z}(t)$ contains two angular rates of Line of Sight, LOS, angular velocity, which reflects two

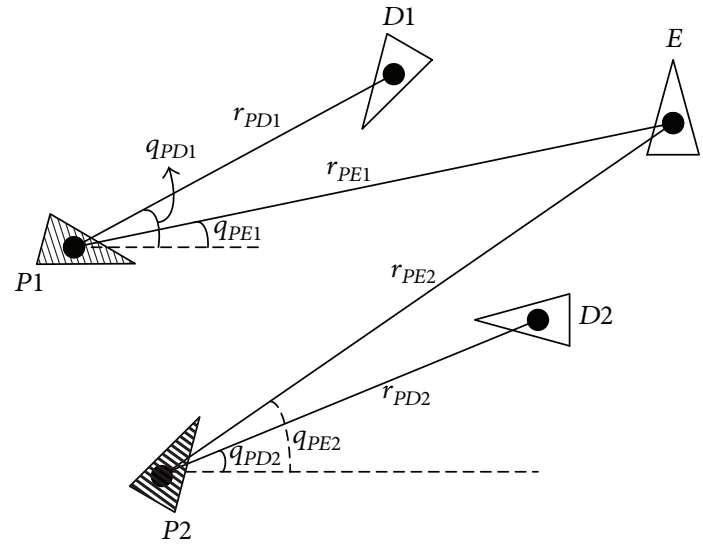

Figure 15: Two Defenders versus two Pursuers scenario.

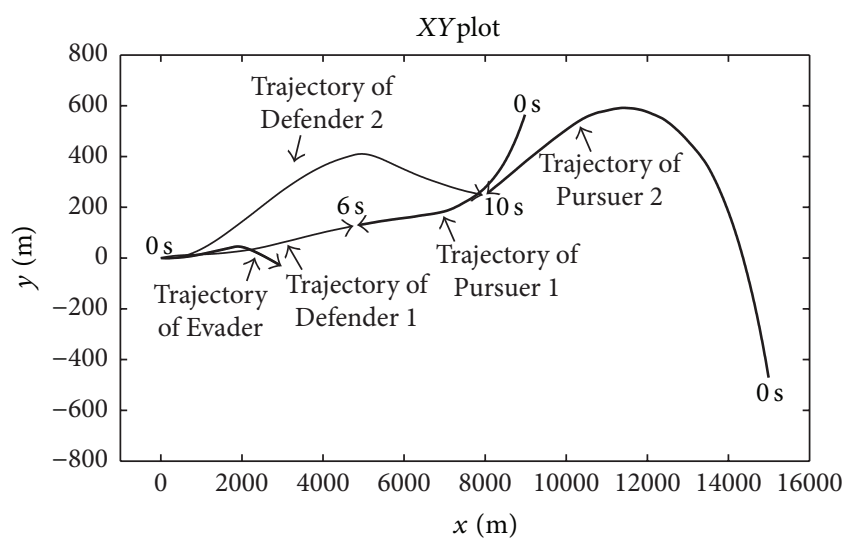

Figure 16: The trajectories of facing two Pursuers.

Defenders' guidance miss-distance. The closed-loop system should satisfy

$$
\mathbf{y}^{T}(t) \mathbf{S}_{z}(t) \mathbf{y}(t) \leq 1, \quad \forall t \in[0, T]
$$

Thus, the chosen weighted matrix $\mathbf{S}_{z}(t)$ is crucial for the guidance design. The weights reflect attention degree of the corresponding LOS angular velocity. $\mathbf{S}_{z}(t)$ may have the form

$$
\mathbf{S}_{z}(t)=\left[\begin{array}{cc}
s_{1}(t) & 0 \\
0 & s_{2}(t)
\end{array}\right],
$$

where $s_{1}(t)$ and $s_{2}(t)$ are both larger than zero. We set an appropriate larger weight for the corresponding Defender whose guidance time-to-go is smaller than the other one. The purpose is to drive the corresponding LOS angular velocity convergence more rapidly. According to the parameters in Table 4, the previously mentioned models, and the methods, we design the corresponding controllers in different stages and add them to the original system. The five aircraft motion trajectories are shown in Figure 16.

It can be seen that, at $0 \mathrm{~s}$, two Pursuers from different directions are guided to the Evader, which simultaneously release two Defenders. Since the distances between the Defender and Evader are not the same, the Evader tends to 
TABLE 4: Simulation parameters of multi-Defender versus multi-Pursuer case.

(a)

\begin{tabular}{lcccccccc}
\hline$V_{P E 1}, V_{P E 2}$ & $V_{P D 1}, V_{P D 2}$ & $t_{f P E 1}$ & $t_{f P D 1}$ & $t_{e P D 1}$ & $t_{f P E 2}$ & $t_{f P D 2}$ & $\mathbf{S}_{w 1}, \mathbf{S}_{w 2}$ & $s_{2}(t)$ \\
\hline $1000 \mathrm{~m} / \mathrm{s}$ & $1500 \mathrm{~m} / \mathrm{s}$ & $9 \mathrm{~s}$ & $6 \mathrm{~s}$ & $5.95 \mathrm{~s}$ & $15 \mathrm{~s}$ & $10 \mathrm{~s}$ & $\mathbf{I}_{1 \times 1}$ & $1000 t$ \\
\hline
\end{tabular}

(b)

\begin{tabular}{cccccccc}
\hline$t_{e P D 2}$ & $\tau_{E}$ & $\tau_{D 1}, \tau_{D 2}$ & $\tau_{P 1}, \tau_{P 2}$ & $N$ & $K$ & $T_{1}$ & $T_{2}$ \\
\hline $9.95 \mathrm{~s}$ & $0.1 \mathrm{~s}$ & $0.05 \mathrm{~s}$ & $0.05 \mathrm{~s}$ & 4 & 2 & $5.95 \mathrm{~s}$ & $9.95 \mathrm{~s}$ \\
\hline
\end{tabular}

(c)

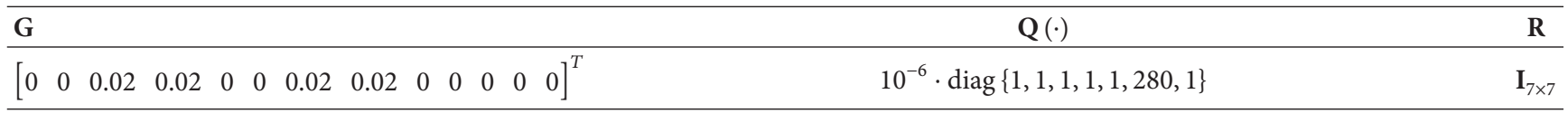

(d)

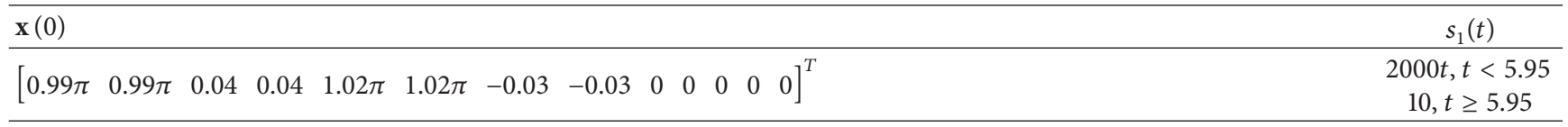

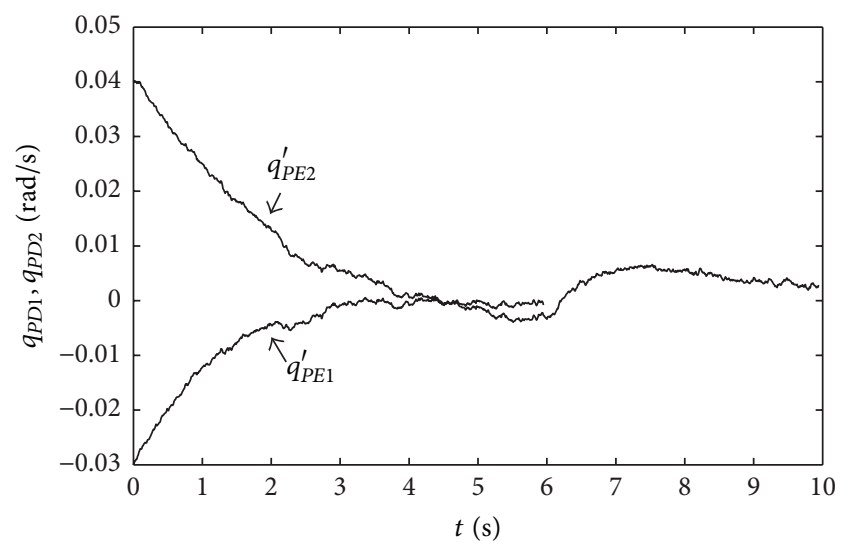

FIGURE 17: The LOS angle velocities between the two Defenders and the two Pursuers.

corporate with the Defender with less guidance time-to-go. After one Defender finishes the guidance process, the Evader turns to fully cooperate with the other Defender.

Figure 17 shows the LOS angle velocities between the two Defenders and the two Pursuers. It can be established that the controller can drive the LOS angle velocities close to zero in the concerned finite-time interval, which means the missdistances satisfied the demand.

Figure 18 presents the Evader's acceleration. It can be established that, before $5.95 \mathrm{~s}$, the Evader is more inclined to cooperate with the Defender with the less guidance timeto-go. After $5.95 \mathrm{~s}$, one Defender has finished the guidance process; the Evader turns to fully cooperate with the other Defender. At the same time, the Evader acceleration does not exceed the physical capacity constraint.

\section{Conclusion}

This paper focuses on a triangle interception scenario; the guidance law design is deduced to a finite-time controller

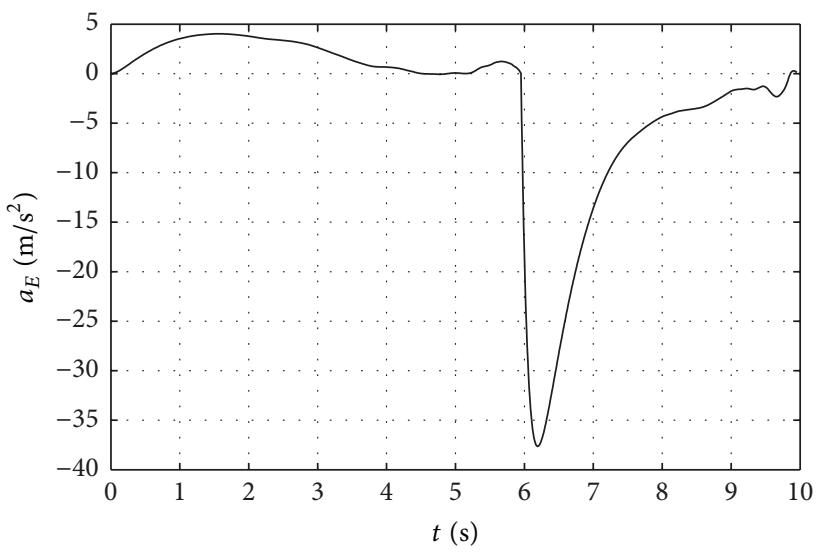

FIGURE 18: The Evader's acceleration.

design problem. Sufficient conditions guaranteeing system of both FTB and IO-FTS are proposed. Then a state feedback controller can be obtained via LMIs. The methods are applied to guidance design for triangle interception scenario. The results show that the proposed method is suitable for solving the problem in a finite-time interval. The method can be regarded as a finite-time stabilization method considering some finite-time performance constraints. Three cases in triangle interception scenarios are studied and the results show that better interception effect will be achieved while Evader and Defender cooperate. In multi-Defender versus multi-Pursuer case, the Evader is more inclined to cooperate with the Defender with the smaller guidance time-to-go. After one Defender finishes the guidance process, the Evader turns to fully cooperate with the other Defender.

\section{Nomenclature}

$a_{P}, a_{E}, a_{D}: \quad$ The acceleration of Pursuer, Evader, and Defender, respectively

$a_{P C}, a_{E C}, a_{D C}$ : The acceleration command of Pursuer, Evader, and Defender, respectively 


\begin{tabular}{|c|c|}
\hline$a_{E \max }:$ & $\begin{array}{l}\text { The physical constraint of Evader } \\
\text { acceleration }\end{array}$ \\
\hline$D:$ & The size of Defender's blind zone \\
\hline$K:$ & $\begin{array}{l}\text { A constant in augmented proportional } \\
\text { navigation (APN) guidance law }\end{array}$ \\
\hline$N:$ & A constant in APN guidance law \\
\hline$q_{P E}, q_{P D}:$ & $\begin{array}{l}\text { The Line of Sight (LOS) angle between } \\
\text { Pursuer and Evader, the LOS angle } \\
\text { between Pursuer and Defender }\end{array}$ \\
\hline$r_{P E}, r_{P D}:$ & $\begin{array}{l}\text { The range between Pursuer and Evader, } \\
\text { the range between Pursuer and } \\
\text { Defender }\end{array}$ \\
\hline$t_{f P E}, t_{f P D}:$ & $\begin{array}{l}\text { The final time of Pursuer-Evader } \\
\text { interception process, the final time of } \\
\text { Pursuer-Defender interception process }\end{array}$ \\
\hline$V_{P E}, V_{P D}:$ & $\begin{array}{l}\text { The approaching speed between } \\
\text { Pursuer and Evader, the approaching } \\
\text { speed between Pursuer and Defender }\end{array}$ \\
\hline $\mathrm{ZEM}_{P D}:$ & $\begin{array}{l}\text { The Zero-Effort-Miss distance of } \\
\text { Defender to the Pursuer }\end{array}$ \\
\hline$\tau_{P}, \tau_{E}, \tau_{D}$ & $\begin{array}{l}\text { The guidance system time constant of } \\
\text { Pursuer, Evader, and Defender, } \\
\text { respectively. }\end{array}$ \\
\hline
\end{tabular}

\section{Conflict of Interests}

The authors declare that there is no conflict of interests regarding the publication of this paper.

\section{Acknowledgment}

This work is supported by National Natural Science Foundation (NNSF) of China under Grants no. 61304239, no. 61304001, and no. 51475462.

\section{References}

[1] R. L. Boyell, "Defending a moving target against missile or torpedo attack," IEEE Transactions on Aerospace and Electronic Systems, vol. 12, no. 4, pp. 522-526, 1976.

[2] L. R. Boyell, "Counterweapon aiming for defence of a moving target," IEEE Transactions on Aerospace and Electronic Systems, vol. 16, no. 3, pp. 402-408, 1980.

[3] A. Ratnoo and T. Shima, "Line of sight guidance for defending an aircraft," in Proceedings of the AIAA Guidance, Navigation, and Control Conference, Toronto, Canada, 2010.

[4] T. Yamasaki and S. N. Balakrishnan, "Triangle intercept guidance for aerial defense," in Proceedings of the AIAA Guidance, Navigation, and Control Conference, Toronto, Canada, August 2010.

[5] V. Shaferman and T. Shima, "Cooperative multiple-model adaptive guidance for an aircraft defending missile," Journal of Guidance, Control, and Dynamics, vol. 33, no. 6, pp. 1801-1813, 2010.

[6] T. Yamasaki, S. N. Balakrishnan, and H. Takano, "Modified CLOS intercept guidance for aircraft defense against a guided missile," in Proceedings of the AIAA Guidance, Navigation, and Control Conference, Portland, Ore, USA, 2011.
[7] A. Ratnoo and T. Shima, "Guidance laws against defended aerial targets," in Proceedings of the AIAA Guidance, Navigation, and Control Conference, Portland, Ore, USA, August 2011.

[8] A. Ratnoo and T. Shima, "Line-of-sight interceptor guidance for defending an aircraft," Journal of Guidance, Control, and Dynamics, vol. 34, no. 2, pp. 522-532, 2011.

[9] T. Shima, "Optimal cooperative pursuit and evasion strategies against a homing missile," Journal of Guidance, Control, and Dynamics, vol. 34, no. 2, pp. 414-425, 2011.

[10] S. Rubinsky and S. Gutman, "Three body guaranteed pursuit and evasion," in Proceedings of the AIAA Guidance, Navigation, and Control Conference, Minneapolis, Minn, USA, August 2012.

[11] A. Ratnoo and T. Shima, "Guidance strategies against defended aerial targets," Journal of Guidance, Control, and Dynamics, vol. 35, no. 4, pp. 1059-1068, 2012.

[12] P. Dorato, "Short-time stability in linear time-varying systems," in Proceedings of the IRE International Convention Record Part 4, vol. 1, pp. 83-87, New York, NY, USA, 1961.

[13] F. Amato, M. Ariola, and P. Dorato, "Finite-time control of linear systems subject to parametric uncertainties and disturbances," Automatica, vol. 37, no. 9, pp. 1459-1463, 2001.

[14] F. Amato and M. Ariola, "Finite-time control of discrete-time linear systems," IEEE Transactions on Automatic Control, vol. 50, no. 5, pp. 724-729, 2005.

[15] S. P. Bhat and D. S. Bernstein, "Finite-time stability of continuous autonomous systems," SIAM Journal on Control and Optimization, vol. 38, no. 3, pp. 751-766, 2000.

[16] W. Chen and L. C. Jiao, "Finite-time stability theorem of stochastic nonlinear systems," Automatica, vol. 46, no. 12, pp. 2105-2108, 2010.

[17] H. Du, C. Qian, S. Yang, and S. Li, "Recursive design of finitetime convergent observers for a class of time-varying nonlinear systems," Automatica, vol. 49, no. 2, pp. 601-609, 2013.

[18] F. Amato, R. Ambrosino, C. Cosentino, and G. De Tommasi, "Input-output finite time stabilization of linear systems," Automatica, vol. 46, no. 9, pp. 1558-1562, 2010.

[19] F. Amato, R. Ambrosino, M. Ariola, C. Cosentino, and G. De Tommasi, Finite-Time Stability and Control, vol. 453 of Lecture Notes in Control and Information Sciences, Springer, London, UK, 2014.

[20] F. Amato, G. Carannante, G. De Tommasi, and A. Pironti, "Input-output finite-time stability of linear systems: necessary and sufficient conditions," IEEE Transactions on Automatic Control, vol. 57, no. 12, pp. 3051-3063, 2012.

[21] F. Amato, G. De Tommasi, and A. Pironti, "Necessary and sufficient conditions for input-output finite-time stability of impulsive dynamical systems," in Proceedings of the American Control Conference (ACC '15), pp. 5998-6003, Chicago, Ill, USA, July 2015.

[22] Y. Guo, Y. Yao, S. Wang, K. Ma, K. Liu, and J. Guo, "Inputoutput finite-time stabilization of linear systems with finitetime boundedness," ISA Transactions, vol. 53, no. 4, pp. 977-982, 2014.

[23] C. Li and Z. Qu, "Distributed finite-time consensus of nonlinear systems under switching topologies," Automatica, vol. 50, no. 6, pp. 1626-1631, 2014.

[24] M. Golestani, I. Mohammadzaman, and A. R. Vali, "Finitetime convergent guidance law based on integral backstepping control," Aerospace Science and Technology, vol. 39, pp. 370-376, 2014. 
[25] M. Golestani and I. Mohammadzaman, "PID guidance law design using short time stability approach," Aerospace Science and Technology, vol. 43, pp. 71-76, 2015.

[26] Y. Guo, S. Wang, Y. Yao, and B. Yang, "Evader maneuver on consideration of energy consumption in flight vehicle interception scenarios," Aerospace Science and Technology, vol. 15, no. 7, pp. 519-525, 2011.

[27] D. Zhou, S. Sun, and K. L. Teo, "Guidance laws with finite time convergence," Journal of Guidance, Control, and Dynamics, vol. 32, no. 6, pp. 1838-1846, 2009.

[28] Y. Guo, Y. Yao, S. Wang, B. Yang, K. Liu, and X. Zhao, "Finite-time control with $H_{\infty}$-infinity constraints of linear time-invariant and time-varying systems," Journal of Control Theory and Applications, vol. 11, no. 2, pp. 165-172, 2013.

[29] Y. Guo, Y. Yao, S. C. Wang, B. Q. Yang, F. H. He, and P. Zhang, "Maneuver control strategies to maximize prediction errors in ballistic middle phase," Journal of Guidance, Control, and Dynamics, vol. 36, no. 4, pp. 1225-1234, 2013.

[30] Y. Guo, X. X. Hu, S. C. Wang, J. Xie, and Q. H. Gao, "Input-output finite-time guidance laws in triangle interception scenario," in Proceedings of the 33rd Chinese Control Conference (CCC '14), pp. 804-809, IEEE, Nanjing, China, July 2014.

[31] O. M. Golan and T. Shima, "Head pursuit guidance for hypervelocity interception," in Proceedings of the AIAA Guidance, Navigation, and Control Conference and Exhibit, Providence, RI, USA, August 2004.

[32] T. Shima and O. M. Golan, "Head pursuit guidance," Journal of Guidance, Control, and Dynamics, vol. 30, no. 5, pp. 1437-1444, 2007. 


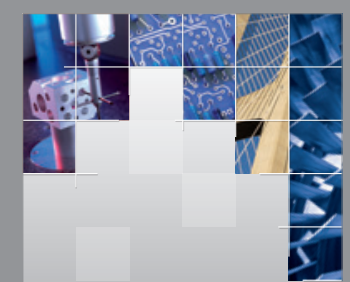

\section{Enfincering}
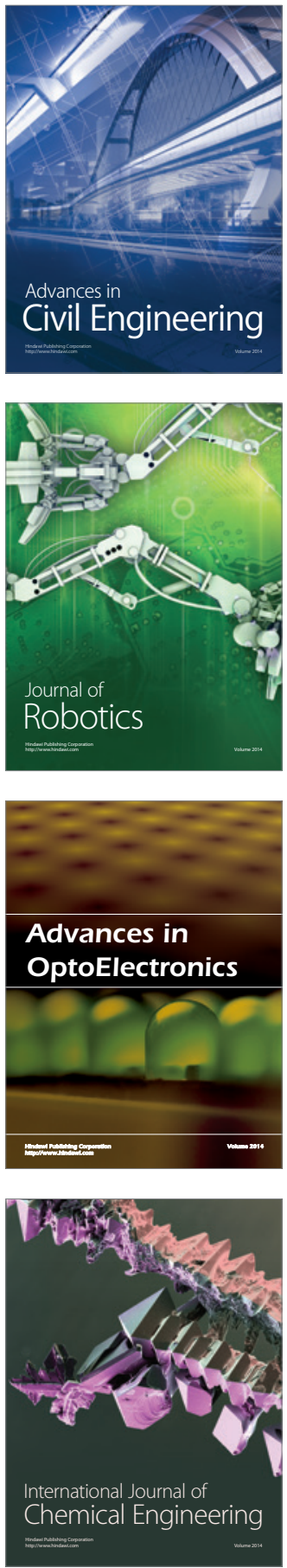

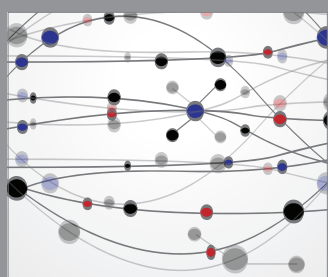

The Scientific World Journal

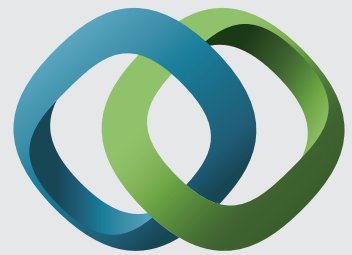

\section{Hindawi}

Submit your manuscripts at

http://www.hindawi.com
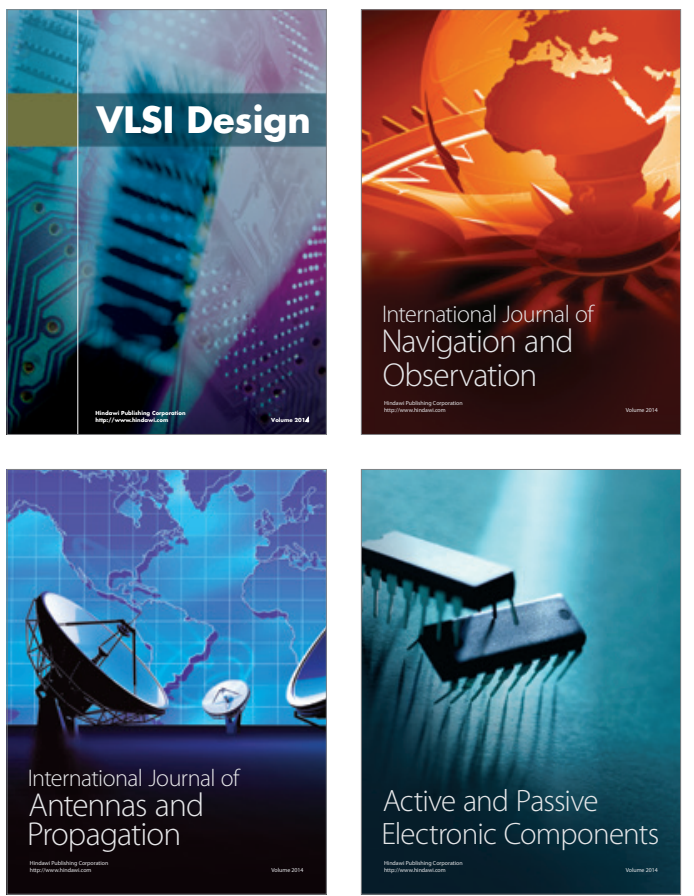
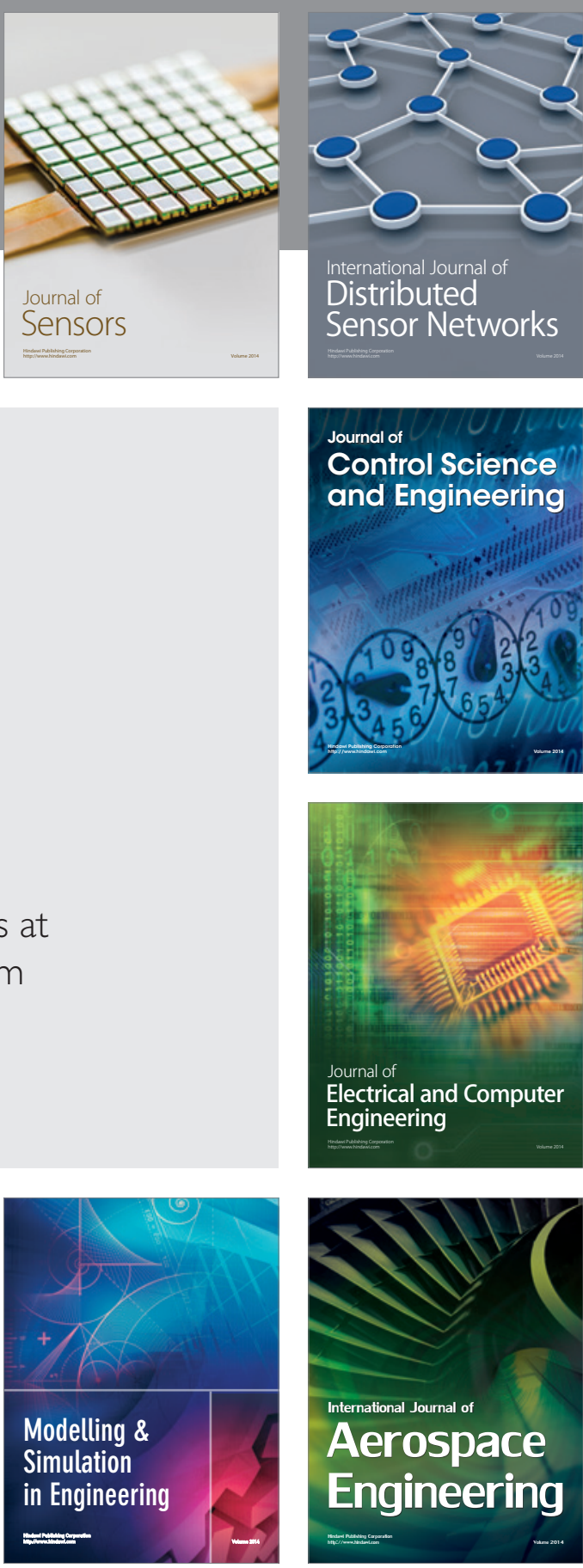

International Journal of

Distributed

Sensor Networks

Journal of

Control Science

and Engineering
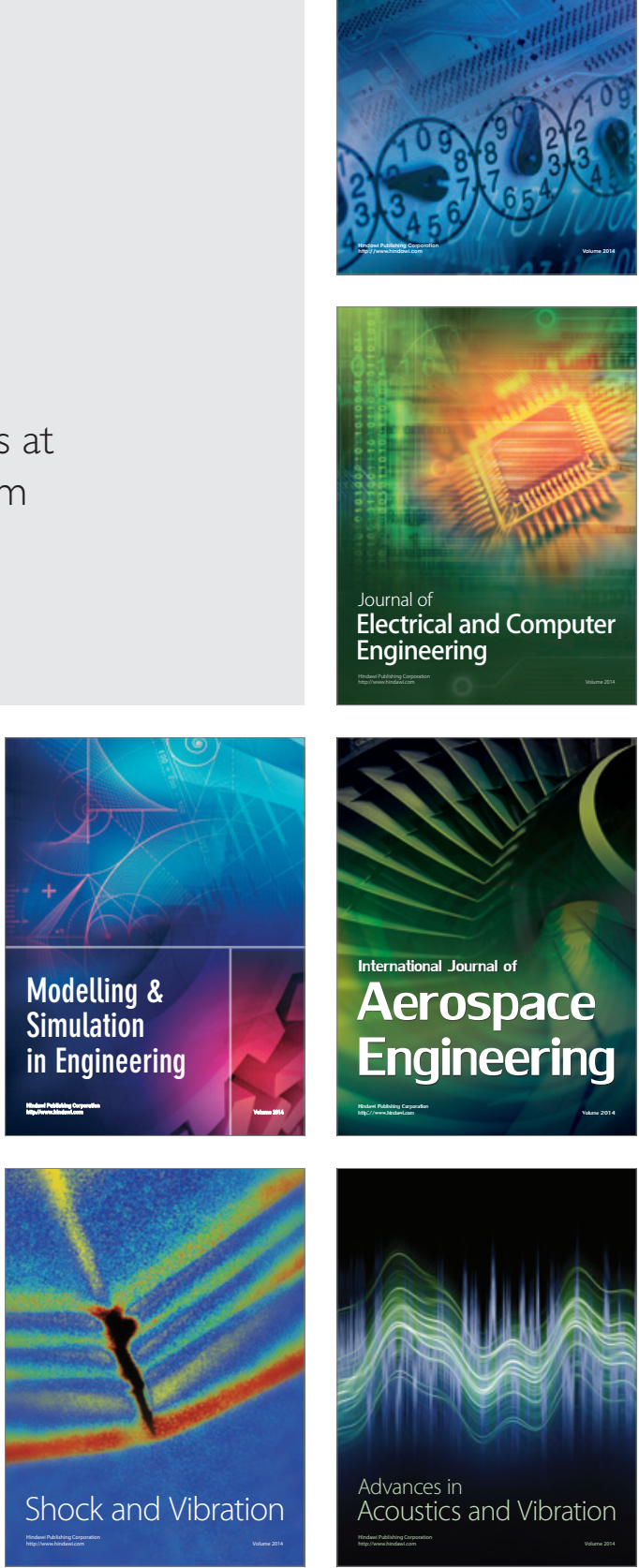